LA W RENCE LIVERM ORE N A TIONAL LABORATORY

Event-by-Event Modeling of Prompt Neutrons and Photons from Neutron-Induced and Spontaneous Fission with FREYA

R. Vogt, J. Randrup

March 1, 2013

ND2013

New York, NY, United States

March 4, 2013 through March 8, 2013 
This document was prepared as an account of work sponsored by an agency of the United States government. Neither the United States government nor Lawrence Livermore National Security, LLC, nor any of their employees makes any warranty, expressed or implied, or assumes any legal liability or responsibility for the accuracy, completeness, or usefulness of any information, apparatus, product, or process disclosed, or represents that its use would not infringe privately owned rights. Reference herein to any specific commercial product, process, or service by trade name, trademark, manufacturer, or otherwise does not necessarily constitute or imply its endorsement, recommendation, or favoring by the United States government or Lawrence Livermore National Security, LLC. The views and opinions of authors expressed herein do not necessarily state or reflect those of the United States government or Lawrence Livermore National Security, LLC, and shall not be used for advertising or product endorsement purposes. 


\title{
Event-by-Event Modeling of Prompt Neutrons and Photons from Neutron-Induced and Spontaneous Fission with FREYA
}

\author{
R. $\operatorname{Vogt}^{1, *}$ and J. Randrup ${ }^{2}$ \\ ${ }^{1}$ Physics Division, Lawrence Livermore National Laboratory, Livermore, CA 94551, USA \\ Physics Department, UC Davis, Davis, CA 95616, USA \\ ${ }^{2}$ Nuclear Science Division, Lawrence Berkeley National Laboratory Berkeley, CA 94720, USA
}

(Dated: February 28, 2013)

\begin{abstract}
The fast event-by-event fission code FREYA generates large samples of complete fission events. Using FREYA, it is possible to obtain the fission products as well as the prompt neutrons and photons emitted during the fission process, all with complete kinematic information. We can therefore extract any desired correlation observables. Concentrating on ${ }^{239} \mathrm{Pu}(n, \mathrm{f}),{ }^{235} \mathrm{U}(n, \mathrm{f})$ and ${ }^{252} \mathrm{Cf}(\mathrm{sf})$, we compare our FREYA results with available data on prompt neutron and photon emission
\end{abstract}

\section{INTRODUCTION}

Our code FREYA (Fission Reaction Event Yield Algorithm) $[1-3,11,12]$. simulates complete fission events with full kinematic information on the fission products and the emitted neutrons and photons. FREYA uses measured observables to improve our understanding of the fission process. Thus it is a potentially powerful tool for bridging the gap between current microscopic models and important fission observables as well as for improving estimates of fission characteristics for applications.

\section{FREYA INPUTS}

We start with a fissile nucleus ${ }^{A_{0}} Z_{0}$ with excitation energy $E_{0}^{*}$ that undergoes binary fission into a heavy ${ }^{A_{H}} Z_{H}$ and a light fragment ${ }^{A_{L}} Z_{L}$. The fragment masses are obtained from experimental mass yields $Y(A)$, see Ref. [2].

Once the mass and charge of the two fragments has been selected, the $Q$ value of the fission channel is the difference between the total mass of $A_{0}$ and the fragment ground-state masses, $Q_{L H}=M\left(A_{0}\right)-M_{L}-M_{H}$. The $Q_{L H}$ value is divided between the total kinetic energy (TKE) and the total excitation energy (TXE) of the fragments. The average TKE is assumed to take the form $\overline{\operatorname{TKE}}\left(A_{H}, E_{\mathrm{n}}\right)=\overline{\operatorname{TKE}}_{\mathrm{data}}\left(A_{H}\right)+d \operatorname{TKE}\left(E_{\mathrm{n}}\right)$. The first term is extracted from data while the second is adjusted to the measured average neutron multiplicity, $\bar{\nu}$.

The fragments acquire angular momentum, $\boldsymbol{S}_{f}$ where $f=L, H$, at scission perpendicular to the line joining the fragment centers. The angular momentum components

\footnotetext{
* Corresponding author: vogt2@llnl.gov
}

are sampled from a statistical distribution with temperature parameter $T_{S}, P\left(S_{f}^{2}\right) \sim \exp \left(-S_{f}^{2} / 2 \mathcal{I}_{f} T_{S}\right)$. where $\mathcal{I}_{f}$ is the moment of inertia of the fragment $f$. We employ half the rigid body value, $\mathcal{I}_{f}=\frac{1}{5} m_{N} r_{0}^{2} A^{5 / 3}$. After the angular momenta are sampled, the rotational energy is $E_{\text {rot }}=\left(\hbar^{2} / 2\right)\left(S_{L}^{2} / \mathcal{I}_{L}+S_{H}^{2} / \mathcal{I}_{H}\right)$. The statistical fragment excitation energy is reduced correspondingly.

After the average total fragment kinetic energy, $\overline{\mathrm{TKE}}$, has been sampled, the combined statistical fragment excitation energy, $\overline{\mathrm{TXE}}$, follows from energy conservation, $\overline{\mathrm{TXE}}=\bar{E}_{L}^{*}+\bar{E}_{H}^{*} \doteq Q-\overline{\mathrm{TKE}}-E_{\mathrm{rot}}$.

If the fragments are in mutual thermal equilibrium, their temperatures are equal, $T_{L}=T_{H}$, and their statistical energy is proportional to the leveldensity parameter, i.e. $\bar{E}_{f}^{*} \sim a_{f}$. FREYA first assigns average excitations based on such equipartition, $\dot{E}_{f}^{*}=a_{f}\left(\tilde{E}_{f}^{*}\right) \overline{\mathrm{TXE}} /\left(a_{L}\left(\tilde{E}_{L}^{*}\right)+a_{H}\left(\tilde{E}_{H}^{*}\right)\right)$. where $\tilde{E}_{f}^{*}=$ $\left(A_{f} / A_{0}\right) \overline{\mathrm{TXE}}$. Subsequently, because the observed neutron multiplicities suggest that the light fragments are more excited (probably due to their greater distortion at scission), the average excitations are adjusted as $\bar{E}_{L}^{*}=$ $x \hat{E}_{L}^{*}, \bar{E}_{H}^{*}=\overline{\mathrm{TKE}}-\bar{E}_{L}^{*}$, where $x>1$ is a parameter.

After the mean excitation energies have been assigned, FREYA accounts for thermal fluctuations. The fragment temperature $T_{f}$ is obtained from $\bar{U}_{f} \equiv U_{f}\left(\bar{E}_{f}^{*}\right)=a_{f} T_{f}^{2}$, where $U\left(E^{*}\right)=E^{*}$. The variance in the excitation $E_{f}^{*}$ is then $\sigma_{f}^{2}=2 \bar{U}_{f}^{*} T_{f}$. Therefore, for each of the two fragments, we sample a thermal fluctuation $\delta E_{f}^{*}$ from a normal distribution of variance $\sigma_{f}^{2}$ and modify the fragment excitation energies as, $E_{f}^{*}=\bar{E}_{f}^{*}+\delta E_{f}^{*}$. Energy conservation causes a compensating fluctuation in TKE leading to $\mathrm{TKE}=\overline{\mathrm{TKE}}-\delta E_{L}^{*}-\delta E_{H}^{*}[3]$.

Neutron evaporation occurs after the fragments have reached their asymptotic velocities. For a fragment of 
statistical excitation $E^{*}$, the maximum temperature in its evaporation daughter, $T_{\max }$, is obtained from $a T_{\max }^{2}=$ $E^{*}-S_{n}(Z, A)$, where $S_{n}(Z, A)$ is the neutron separation energy. The neutron kinetic energy $\epsilon$ is sampled from $f_{n}(\epsilon) \sim \epsilon \exp \left(-\epsilon / T_{\max }\right)$. The emitted neutron is assumed to carry no angular momentum so the fragment angular momentum is unaffected by neutron emission. Neutrons are emitted as long as the $Q$ value for emission exceeds $E_{n, \text { cut }}$ where photon emission takes over.

After neutron evaporation has ceased, the residual product nucleus has a statistical excitation energy of $E^{*}<S_{n}(Z, A)+E_{n, \text { cut }}$ and de-excites by sequential photon emission. First the statistical excitation energy is radiated away by sequential photon emission, leaving a cold but rotating product nucleus which then emits photons along the yrast line.

Statistical photon emission is treated analogous to neutron evaporation except there is no separation energy for photons. Since the photons are massless, we introduce an infrared cut-off energy. Furthermore, there is an extra energy factor in the photon phase space, $f_{\gamma}(E) \sim E^{2} \exp (-E / T)$ where $T$, nuclear temperature prior to emission is equal to the maximum possible temperature after emission. Photons are emitted isotropically in the frame of the emitter nucleus. Emission continues until the available statistical excitation energy has been exhausted. The angular momentum is then disposed of by simulating a stretched E2 cascade for as long as $S>2$. Each photon emission reduces the angular momentum by two units and the energy by $E=(1 / 2)\left[S^{2}-(S-2)^{2}\right] \hbar^{2} / \mathcal{I}=2(S-1) \hbar^{2} / \mathcal{I}$. At the end of the cascade, when $S<2$, a single final photon is emitted with the remaining excitation energy.

\section{NEUTRON RESULTS}

We first present some neutron observables from fission of ${ }^{252} \mathrm{Cf}(\mathrm{sf})$ and ${ }^{239} \mathrm{Pu}(n, \mathrm{f})$ in Fig 1 .

The average neutron kinetic energy obtained from FREYA is compared to the Tsuchiya data [4] on ${ }^{239} \mathrm{Pu}(n, \mathrm{f})$ in the top panel of Fig. 1. While the general trends are similar, FREYA overestimates the neutron kinetic energy near $A \approx 110$ and underestimates it near $A \approx 125$.

The second from top panel of Fig. 1 presents measurements of $\nu(\mathrm{TKE})$ in ${ }^{252} \mathrm{Cf}(\mathrm{sf})$. Bowman [5] extracted $\operatorname{TKE}(A)$, but did not simultaneously measure the fragment yields. Thus the average neutron multiplicity depends only on $\operatorname{TKE}(A)$. Budtz-Jørgensen [6] measured both $Y(A)$ and $\operatorname{TKE}(A)$ so that $\nu(\mathrm{TKE})$ represents an average over both quantities. FREYA and FIFRELIN [7], both shown with the data, also account for $Y(A)$ and $\operatorname{TKE}(A)$ and thus agree well with Budtz-Jørgensen [6].

The third from top panel of Fig. 1 compares the neutron multiplicity distribution $P(\nu)$ for ${ }^{239} \mathrm{Pu}(n, \mathrm{f})$ from FREYA to the compilation of Holden and Zucker [8]. Both results are considerably different from a Poisson because each neutron removes not only its kinetic energy but its separation energy while the Poisson only accounts for kinetic energy.

The observable $\nu(A)$ is very sensitive to the division of TXE between fragments, governed by the parameter $x$ in FREYA. The characteristic 'sawtooth' behavior is well reproduced by FREYA, as shown in the bottom panel of Fig. 1. There is a minimum in $\nu(A)$ near $A_{H} \sim 132$, where $\operatorname{TKE}\left(A_{H}\right)$ is maximized [11]. Due to the closed shell at $A=132$, the fragments are particularly resistant to neutron emission.

\section{PHOTON RESULTS}

Here FREYA results are compared to photon data spanning the last four decades, all taken on ${ }^{252} \mathrm{Cf}(\mathrm{sf})$. For further details, see Ref. [12].

The top panel of Fig. 2 compares FREYA results on average total photon energy as a function of $A, E_{\gamma}(A)$, to data [14]. There is a sharp drop in the measured $E_{\gamma}$ at symmetry, $A=126$, while FREYA shows a dip near $A=132$, similar to $\nu(A)$. While the statistics are rather poor, results for $T_{S}=0.35$ and $0.75 \mathrm{MeV}\left(\bar{S}_{f} \sim 3.9 \hbar\right.$ and $5.8 \hbar$ respectively) are, on average, too low to reproduce the data. The results for $T_{S}=2.75\left(\bar{S}_{f} \sim 11 \hbar\right)$ and $T_{S}=0.2 \mathrm{MeV}\left(\bar{S}_{f} \sim 3 \hbar\right.$ with $\left.E_{n \text { cut }}=1 \mathrm{MeV}\right)$ are rather similar for the light fragment but differ for the heavy fragment.

The average photon energy decreases with TKE, as seen in the top right panel of Fig. 2. The Nardi data [14] starts out at lower $\bar{E}_{\gamma}$, becoming almost independent of TKE for TKE $>180 \mathrm{MeV}$. The FREYA result with $E_{n, \text { cut }}=0.01 \mathrm{MeV}$ and $T_{S}=2.75 \mathrm{MeV}$ is in relatively good agreement with this data at high TKE. The linear decrease of the Nifenecker data [13] with TKE agrees with neither the Nardi data nor FREYA. A new measurement of $\bar{E}_{\gamma}(A)$ and $\bar{E}_{\gamma}(\mathrm{TKE})$ would be very helpful for resolving ssuch discrepancies in the data. Note also that the dependence of $\bar{E}_{\gamma}(\mathrm{TKE})$ in FREYA is quite different than $\nu$ (TKE), as shown in the second from top panel of Fig. 1.

The data of both Nardi [14] and Nifenecker [13], while differing in detail, support a rather high value of fragment spin, $\sim 11 \hbar$. However, the more recently DANCE photon multiplicity [16] is consistent with a lower value, $\bar{S}_{f} \sim$ 3.9ћ. Calculations with both $\bar{S}_{f}$ are compared to the data, along with the Poisson result, in the third from top panel of Fig. 2. The resolution of the difference between the new and old data is important.

The LiBerACE data on neutron-photon correlations are compared to FREYA calculations with $T_{S}=0.35$ and $2.75 \mathrm{MeV}$ in the bottom panel of Fig. 2. Niefenecker claimed a strong positive neutron-photon correlation [13]. The LiBerACE data [17] instead show only a weak correlation. FREYA produces a slight anticorrelation, as might be expected from simple conservation laws. The lower value of $T_{S}$ gives both a lower multiplicity and a stronger negative shift between $\nu=2$ and $\nu=4$ than the data. 

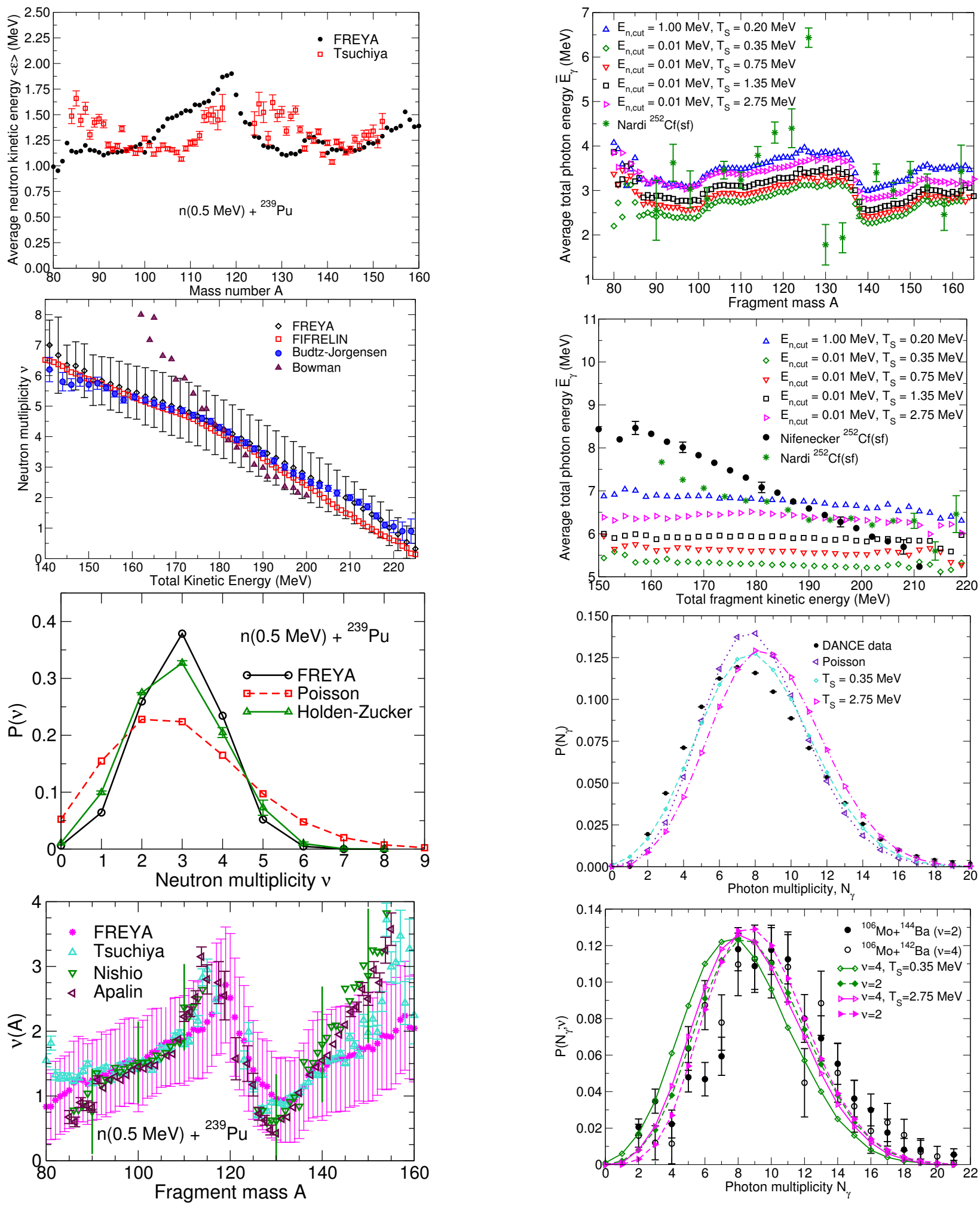

FIG. 1. (Top) Average neutron kinetic energy for ${ }^{239} \mathrm{Pu}(n, \mathrm{f})$ compared to data [4]. (Second from top) Average ${ }^{252} \mathrm{Cf}(\mathrm{sf})$ $\nu$ (TKE) measured by Budtz-Jørgensen [6] and Bowman [5] compared to FREYA (including variance) and FIFRELIN [7]. (Third from top) Neutron multiplicity distribution, $P(\nu)$, for ${ }^{239} \mathrm{Pu}(n, \mathrm{f})[8]$ compared to FREYA and a Poisson distribution. (Bottom) Average $\nu(A)$ for ${ }^{239} \mathrm{Pu}(n, \mathrm{f})$. The FREYA results [11] (including variance) are compared to data $[4,9,10]$.

FIG. 2. Photon emission from ${ }^{252} \mathrm{Cf}(\mathrm{sf})[12]$. (Top) $E_{\gamma}(A)$ compared to data [14]. (Second from top) $E_{\gamma}$ (TKE) compared to data from Niefenecker [13] and Nardi [14]. (Third from top) The photon multiplicity [16] compared to FREYA calculations and a Poisson with $T_{S}=0.35 \mathrm{MeV}(\triangleleft)$. (Bottom) The $N_{\gamma}$ distribution gated on $\nu$, averaged over all fragment masses. The solid curves with filled symbols show $\nu=2$ while the dashed curves with open symbols show $\nu=4$. The LiBerACE [17] Mo+Ba data with $\nu=2$ and 4 are also shown. 


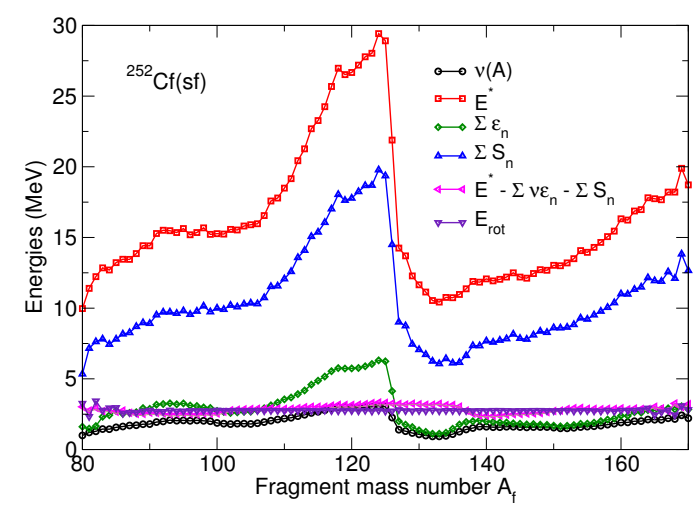

FIG. 3. The ways $E^{*}$ is expended during deexcitation.

\section{COMPONENTS OF THE FRAGMENT EXCITATION ENERGY}

The measured photon observables exhibit an $A$ dependence similar to $\bar{\nu}(A)$. However, our photon results are almost independent of both $A$ and TKE, unlike the calculated $\bar{\nu}(A)$, see Figs. 1 and 2. Such independence can be expected because the product excitation energy is fairly independent of $E^{*}$ for residual excitations less than $S_{n}$.

Figure 3 shows how the initial statistical excitation energy, $E^{*}$, is used up. Most of it is used to remove the evaporated neutrons, $\sum_{i=1}^{\nu} S_{n}(Z, A-i+1)$ where $\nu$ is the neutron multiplicity (which varies event-by-event), and also has a sawtooth form. The evaporated neutron kinetic energy is several times smaller than $S_{n}$. Thus $\sum_{i=1}^{\nu} \epsilon_{i}$ is smaller but also sawtooth-like.

The statistical excitation energy in the residual product nucleus is then (apart from recoil effects) $E^{*}-$ $\sum_{i=1}^{\nu}\left[S_{n}(Z, A-i+1)+\epsilon_{i}\right]$. This quantity does not have any noticeable sawtooth structure.

After statistical emission, only $E_{\text {rot }}$ remains. Since we assume that the average $E_{\text {rot }}$ at scission depends smoothly on the mass partition, the average yrast energy and photon multiplicity also depends smoothly on $A$. If photon emission occurs only after neutron emission, the maximum possible $\bar{E}_{\gamma}$ per fragment cannot significantly exceed $S_{n}$ unless $E_{\text {rot }}$ is large. The effect on $\bar{N}_{\gamma}$ is small because increasing $E_{\text {rot }}$ increases $\bar{N}_{\gamma}$ only by the yrast multiplicity, $\sim 1-2$. Thus, $\bar{E}_{\gamma}(A)$ arises from momentum and energy conservation.

Event-by-event Monte Carlo calculations [7, 12, 18] may have difficulties reproducing the structure of the photon data. Because the photons appear at the end of a complicated process, it is not straightforward to "tune" the resulting $A$ dependence in such treatments while still maintaining agreement with other data and conserving the energy and momentum in each event. While other methods of excitation energy partition can reproduce $\bar{\nu}(A)$ more closely, this does not necessarily guarantee good agreement with earlier photon results [13-15].

Some calculations can reproduce the trend of the $\nu(A)$ and $E_{\gamma}(A)$ data [20]. Reference [19] converts the similarity of $E_{\gamma}\left(E_{n}\right)$ and $\bar{\nu}\left(E_{n}\right)$ into a more general relation, $E_{\gamma} \propto \bar{\nu}$. In Refs. [20, 21], this relation is hardwired in and assumed to hold for $E_{\gamma}(A)$ and $\nu(A)$. They also use average values of the neutron separation energies and assume only a limited number of fragment pairs [21] and are thus not true event-by-event calculations.

\section{SUMMARY}

The event-by-event nature of FREYA allows detailed studies of fission observables. FREYA agrees relatively well with most neutron observables [11]. Comparison with existing photon data, on the other hand, do not present a very clear picture. While there agreement with some trends, there are still significant differences in detail [12]. In future work, we will address neutron-photon competition in more detail.

The work of R.V. was performed under the auspices of the U.S. DOE by LLNL under Contract DE-AC5207NA27344. The work of J.R. was performed under the auspices of the U.S. DOE by LBNL under Contract DEAC02-05CH11231.
[1] R. Vogt et al., Phys. Rev. C 80, 044611 (2009).

[2] J. Randrup and R. Vogt, Phys. Rev. C 80, 024601 (2009).

[3] R. Vogt et al., Phys. Rev. C 85, 024608 (2012).

[4] C. Tsuchiya et al., J. Nucl. Sci. Technol. 37, 941 (2000).

[5] H. R. Bowman et al., Phys. Rev. 129, 2133 (1963).

[6] C. Budtz-Jørgensen and H.-H. Knitter, Nucl. Phys. A 490, 307 (1988).

[7] O. Litaize and O. Serot, Phys. Rev. C 82, 054616 (2010).

[8] N. E. Holden and M. S. Zucker, BNL-NCS-35513 (1985).

[9] K. Nishio et al., J. Nucl. Sci. Technol. 32, 404 (1995).

[10] V. F Apalin et al., Nucl. Phys. A 71, 553 (1965).

[11] R. Vogt and J. Randrup, Phys. Rev. C 84, 044621 (2011).

[12] R. Vogt and J. Randrup, submitted to Phys. Rev. C.

[13] H. Nifenecker et al., Nucl. Phys. A 189, 285 (1972).
[14] E. Nardi, A. Gavron, and Z. Fraenkel, Phys. Rev. C 8, 2293 (1973).

[15] F. Pleasonton, R. L. Ferguson, and H. W. Schmitt, Phys. Rev. C 6, 1023 (1972).

[16] A. Chyzh et al., Phys. Rev. C 85, 02160(R) (2012).

[17] D. L. Bleuel et al., Nucl. Instr. Meth. A 624, 691 (2010).

[18] S. Lemaire, P. Talou, T. Kawano, M. B. Chadwick, and D. G. Madland, Phys. Rev. C 73, 014602 (2006).

[19] J. Fréhaut, IAEA-INDC (NDS) 220, 99 (1989).

[20] A. Tudora, C. Morariu, F.-J. Hambsch, S. Oberstedt and C. Manalilescu, Phys. Procedia 31, 43 (2012).

[21] F.-J. Hambsch, S. Oberstedt, A. Tudora, G. Vladuca and I. Ruskov, Nucl. Phys. A 726, 248 (2003). 